Commun. math. Phys. 5, 88-96 (1967)

\title{
Local Fields with the Wrong Connection Between Spin and Statistics*
}

\author{
R. F. Streater \\ Department of Physics, Imperial College, London, S.W. 7
}

Received October 10, 1966

\begin{abstract}
It is proved that there exist free field operators which satisfy local commutativity and which transform according to certain unitary representations of the homogeneous Lorentz group. The fields satisfy axioms similar to the Wightman axioms, and give rise to local algebras of observables obeying postulates similar to those suggested by $\mathrm{H}_{\mathrm{AAG}}$. They describe a tower of particles with spins $\frac{1}{2}, 1 \frac{1}{2}$, $2 \frac{1}{2}, \ldots$, but commute at space-like separation, giving rise to Bose statistics for the particles. This shows that the well-known theorem on spin and statistics cannot be extended to general theories of local observables; it also shows that the assumptions made in "S-matrix theory" do not hold for the $S$-matrix of a theory of interacting infinite fields.
\end{abstract}

\section{Introduction}

There has been some interest lately [1] in theories of elementary particles in which the particles fall into infinite sets which transform as a unitary representation of a non-compact symmetry group. This naturally led to the question of whether the theory can be described by a causal quantized field which transforms in the same way. The work of FELDMAN and MATTHEWs shows that for such fields the spin-statistics theorem of ordinary field theory is no longer valid; in particular, one can quantize a system of particles with spins $\frac{1}{2}, 1 \frac{1}{2}, 2 \frac{1}{2}, \ldots$, with Bose statistics, and still describe them by a local field (in the more general sense).

On the other hand it has been conjectured that the spin-statistics theorem can be proved within the more general framework of the theory of localized observables $[3,4]$. It is therefore worthwhile to give a proof of the existence of a concrete counter-example to this conjecture, by establishing some results similar to the work of [2] on a rigorous basis. This paper is devoted to this end.

* The research reported in this document has been sponsored in part by the Air Force Office of Scientific Research OAR through the European Office Aerospace Research U.S. Air Force. 
The first problem is to construct a realization of the representation of the Poincaré group, $\mathscr{P}$, in a space of functions that transform in a manifestly covariant way. This can be achieved for any representation of the Poincaré group with a specified mass by using a technique exploited by Joos [5] and later by Pursey [6] and Weinberg [7]. We run through this construction in the next section, for an infinite multiplet, all with the same mass. For a finite dimensional representation of the Lorentz group the covariant wave-functions are labelled $\psi_{\alpha}(p)$, and transform as $\psi_{\alpha}(p) \rightarrow S_{\alpha \beta}{ }^{\prime}(\Lambda) \psi_{\beta}\left(\Lambda^{-1} p\right)$ under the action of the Lorentz group. It is more convenient to define the wave-functions in a manner independent of the basis, as follows: let $K$ be a Hilbert space carrying a unitary representation $W: \Lambda \rightarrow W(\Lambda)$ of the Lorentz group $L_{+}^{\uparrow}$; let $p$ be a typical point on the mass-hyperboloid $M_{m}: p^{2} \equiv p_{0}{ }^{2}-\mathbf{p}^{2}=m^{2} ; p_{0}>0$. Then a manifestly covariant wave-function is a map $p \rightarrow \sigma(p) \in K$ which transforms under the Lorentz group according to

$$
\sigma(p) \rightarrow W(\Lambda) \cdot \sigma\left(\Lambda^{-1} p\right) .
$$

We say a wave-function is normalizable if

$$
\int(\sigma(p), \sigma(p)) d \Omega_{p}<\infty
$$

where $d \Omega_{p}$ is the Lorentz-invariant measure $\frac{d^{3} p}{2\left(m^{2}+\mathbf{p}^{2}\right)^{\frac{1}{2}}}$. We shall find it convenient to regard a wave-function in its dual rôle; namely, for each $p \in M_{m}, \sigma$ determines a continuous linear functional $\sigma(p)$ on $K$, thus: $\sigma_{p}(\Psi)=(\sigma(p), \Psi)$. In other words, a wave function determines a map $K \times M_{m} \rightarrow C$.

The normalizable wave-functions form a Hilbert space $\bar{K}$ in which the action of the Lorentz group is unitary. This representation can be extended to a unitary representation $U$ of $\mathscr{P}$ in the same space $\mathscr{K}$ by the action

$$
(U(a) \sigma)(p)=e^{i p a} \sigma(p) .
$$

The space $\widetilde{K}$ is the space of one-particle states. In section 3 we define creation and annihilation operators on the Fock space constructed from $K$ with Bose statistics, and from them construct a free quantized field with the wrong connection between spin and statistics. By "quantized field" we have in mind the following generalization of the Wightman axioms: Let $\mathscr{U}(a, \Lambda)$ be a representation of $\mathscr{P}$ in a Hilbert space $h$ (the space of the states of theory). Let $W_{0}(\Lambda)$ be a representation of $L \uparrow$ in a Hilbert space $K_{0}$ (the auxiliary space of [2]). Let $S$ be the space of testfunctions on $\mathbb{R}^{4}$ of rapid decrease. Then a quantized field is a map $A$ from $K_{0} \times S$ to unbounded operators on $h$ with the usual WightmaN [8] properties: there exists a unique vector $\Psi_{0} \in h$ invariant under $\mathscr{U}$, and

7 Commun. math. Phys., Vol. 5 
a dense domain $D$ invariant under $\mathscr{U}$, and under the action of the field operators:

$A(\alpha, f) D \subset D$ for all $\alpha \in K_{0}, f \in S$ and $\Psi \in D$. Moreover, for each $\Phi \in D$ and $\Psi \in D$ the map $K_{0} \times S \rightarrow C$ given by

$$
(\alpha, f) \rightarrow(\Phi, A(\alpha, f) \Psi)
$$

is separately continuous in $\alpha$ (in the strong topology of $K_{0}$ ) and $f$ (in the usual Schwartz topology). Covariance is expressed in the form

$$
\mathscr{U}(a, \Lambda) A(\alpha, f) \mathscr{U}(a, \Lambda)^{-1}=A\left(W_{0}(\Lambda) \alpha, f_{a, \Lambda}\right)
$$

and causality takes the usual form

$\left[A(\alpha, f), A(\beta, f)^{*}\right]=0$ if the supports of $f$ and $g$ are space-like separated. Moreover, $\mathscr{U}(a, \Lambda)$ is not to possess any subrepresentations with negative energy.

It is in this sense that we construct a quantized field. The Wightman functions satisfy the growth conditions discussed by Borchers and Zimmermans [9] which is sufficient to show that the field is essentially self-adjoint on $D$, and that a theory of localized observables can be set up, according to the argument of ref. [9]. This demonstrates that the hypotheses of references 3 and 4 are insufficient to prove a spin-statistics theorem.

We can naturally define fields transforming according to infinitedimensional non-unitary representations of $L_{+}^{\uparrow}$ by combining the unitary representations with the usual finite-dimensional representations, and reducing the tensor product to its irreducible components. The axioms are easily adjusted to accommodate these. In this way, towers of spins $0,1,2, \ldots$ can be quantized with Fermi-Dirac statistics. One can also quantize these towers with the correct connection between spin and statistics. In this case the concept of localization differs from the one obtained by finite dimensional fields, which are not local relative to the new fields. This shows that non-isomorphic theories of local observables can give rise to the same $S$-matrix (unity in this case).

\section{Manifestly Covariant Form}

In this section we recall the argument of Feldman and Matthews, at the same time fixing the notation.

Let $[j, m]$ be the representation of the Poincaré group with spin $j$ and mass $m$, and let $U_{0}$ be the direct sum of the odd spin representations, all with mass $m$; that is

$$
U_{0}=\underset{j=0}{\oplus}\left[j+\frac{1}{2}, m\right]
$$

where each enters with infinite multiplicity. Let $U$ be the direct sum of $U_{0}$ with itself,

$$
U=U_{0} \oplus U_{0} \text {. }
$$


We denote the carrier space of $U$ by $\mathscr{\mathscr { H }}$, which may thus be written as the direct sum of two isomorphic subspaces, each carrying the representation $U_{\mathbf{0}}$. Vectors in the first space we call particles and vectors in the second, anti-particles. The space $\tilde{\mathscr{H}}$ itself contains all possible states of one particle or anti-particle.

For each $p$ we define the little group $H_{p}$ to be the subgroup of $L_{+}^{\uparrow}$ leaving $p$ fixed. This group acts on space-time, the four-dimensional translation group $T_{4}$, in the usual way; the semi-direct product $G_{p}$ of $H_{p}$ with $T_{4}$ is called the inhomogeneous little group of $p$. If $m^{2}>o, H_{p}$ is isomorphic to $O_{3}$. Let $D^{j}$ be the usual representation of $O_{3}$ with spin $j$, and let $V_{0}$ be the direct sum of $D^{j}$ for odd $2 j$ :

$$
V_{0}=\oplus_{j=0}^{\infty} D^{j+\frac{1}{2}}
$$

where each enters with infinite multiplicity. This is a two-valued representation. Let $V$ be the direct sum of $V_{0}$ with itself

$$
V=V_{0} \oplus V_{0} \text {. }
$$

Let $\mathscr{H}$ be the carrier space of $V$. Then $V$ can be extended to a representation of the inhomogeneous little group of $p$, in $\mathscr{H}$, as follows:

$$
\psi \stackrel{(a, R)}{\longrightarrow} e^{i p a} V(R) \psi
$$

where $p^{2}=m^{2}, \psi \in \mathscr{H}, R \in O_{3}=H_{p}$. It is known [10] that $U$ is the representation of $\mathscr{P}$ induced by this representation. This means that $U$ can be realized as follows. We consider functions on $X=\mathscr{P} / G_{p_{0}}$ for some $p_{0} . X$ is homogeneous with respect to $G_{p_{0}}$ and can be identified with $M_{m}$, the positive-energy branch of the mass hyperboloid. For each $p \in X$, choose the pure Lorentz transformation $L_{p}$ such that $L_{p} p=p_{0}$. Let $F$ be any Hilbert bundle with fibre $\mathscr{H}$ and base-space $X$. We identity vectors in $\mathscr{H}$ with cross-sections of $F$; that is, an element of $\mathscr{\mathscr { H }}$ is a map $\Psi$ from $X$ to $\mathscr{H}$. The Haar measure of $\mathscr{P}$ induces the natural invariant measure $d \Omega_{p}$ in $X$; the scalar product in $\tilde{\mathscr{H}}$ is given by

$$
(\Phi, \Psi)=\int(\Phi(p), \Psi(p)) d \Omega p .
$$

The action of $U$ in $\stackrel{m}{\mathscr{H}}$ is given by

$$
(U(a, \Lambda) \Psi)(p)=e^{i p a} V\left(L_{p} \Lambda L_{\Lambda p^{-1}}^{-1} \Psi\left(\Lambda^{-1} p\right) .\right.
$$

It is easily seen that $L_{p} \Lambda L_{\Lambda p^{-1}} \in H_{p_{0}}$. If $p_{0}=(m, o, o, o)$ the space $\mathscr{H}$ contains the "rest states" of MatThews and Feldman, and $V\left(L_{p}\right)$ is the "boost operator" giving the particles momentum $p$.

We now proceed to introduce the "auxiliary group", chosen to be $S L(2, C)$, and the "auxiliary space", as in ref. [2]. 
It is well-known [11] that there exist unitary representations of $S L(2, C)$ which, restricted to $S U_{2}$, are unitary equivalent to $V$. In the next section we define such a representation, $W$, acting in a space $K$. Corresponding to the reduction $V=V_{0} \oplus V_{0}$, we may reduce $W$ according to $W=W_{0} \oplus W_{0}$ and $K=K_{1} \oplus K_{2}$ where $W_{0}$ is unitary equivalent to $V_{0} . K_{1}$ is the auxiliary space for the particles and $K_{2}$ that for the antiparticles, in the rest frame.

Let $G$ be a Hilbert bundle with base $X$ and fibre $K$. Cross-sections of $G$ form a Hilbert space $\widetilde{K}=\int K(p) d \Omega_{p}$, and $\stackrel{M}{K}$ carries a representation of $\mathscr{P}$, called $W(a, \Lambda)$, defined by

$$
(W(a, \Lambda) \sigma)(p)=e^{i p a} W(\Lambda) \sigma\left(\Lambda_{p}^{-1}\right) .
$$

(Recall that a vector in $\bar{K}$ is a map $\sigma: X \rightarrow K$ such that

$$
\int d \Omega_{p}(\sigma(p), \sigma(p))_{K}<\infty \text {. }
$$

The point of this construction is that $W(a, \Lambda)$ is unitary equivalent to the given representation of $\mathscr{P}, U(a, \Lambda)$. The proof is easy, and is omitted.

\section{Creation and Annihilation 0perators}

The procedure known as second quantization of this representation of $\mathscr{P}$ consists in finding operators $a(\sigma, f)$ for each $\sigma \in K$ and $f \in S$, satisfying the canonical commutation relations

$$
\begin{aligned}
{[a(\sigma, f), a(\varrho, g)] } & =0 \\
{\left[a(\sigma, f), a(\varrho, g)^{*}\right] } & =(\varrho, \sigma) \int \tilde{g}(-p) \tilde{f}(p) \delta\left(p^{2}-m^{2}\right) \theta\left(p_{0}\right) d^{4} p
\end{aligned}
$$

We interpret $a(\sigma, f)^{*}$ as the creation operator for a state vector in $\dddot{K}$ consisting of particles if $\sigma \in K_{1}$ and anti-particles if $\sigma \in K_{2}$. In fact, $a(\sigma, f)^{*}$ creates the vector $\Psi$ in $\widehat{K}$ given by the map $X \rightarrow K$ :

$$
\Psi(p)=f(p) \sigma .
$$

Since the creation and annihilation operators are unbounded operators, these commutation relations must be discussed with the usual caution. In fact, we shall define the Fock representation of these operators; they are defined on a common dense domain $D$ invariant under the action of the operators themselves, and on which they are essentially self-adjoint. The commutation relations are then understood to hold on $D$.

We require that $a(\sigma, f)$ transform under the Poincaré group according to

$$
a(\sigma, f) \stackrel{\mathscr{U}(a, \Lambda)}{\longrightarrow} a\left(W(\Lambda) \sigma, f_{a, \Lambda}\right)
$$

where $f \rightarrow f_{a, \Lambda}$ is the natural action of $\mathscr{P}$ on the test-function space. It follows that $a(\sigma, f)^{*}$ transforms in the same way. This transformation law holds in the Fock representation, defined in the usual way [12]. 
Briefly, define the $n^{\text {th }}$ symmetrized power $\breve{K}^{n}$ of $\dddot{K}$ to be the Hilbert

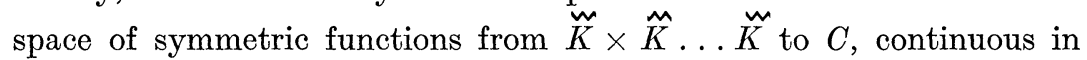
each variable with the remaining held fixed. Then Fock space is the direct sum

$$
h=\sum_{n=0}^{\infty} \oplus \stackrel{m}{K^{n}}
$$

where $\widetilde{m}^{0}$ is the one-dimensional Hilbert space containing the vacuum $\Psi_{0}$. Clearly, $h$ carries a representation $\mathscr{U}$ of $\mathscr{P}$, the direct sum of the identity on $\widetilde{K}^{0}$ and the $n^{\prime}$ th product of $W(a, \Lambda)$ with itself on $\widetilde{K}^{n}$, partially reduced by symmetry. For the domain $D$ we may take the linear span of the set of states with a total of $n$ particles, represented by a vector in $\widetilde{K}^{n}$ of the special form $a\left(\sigma_{1}, f_{1}\right)^{*} \ldots a\left(\sigma_{n}, f_{n}\right) * \Psi_{0}$. We define the operator $a(\sigma, f)$ as follows:

$$
\begin{gathered}
(a(\sigma, f))^{n}\left(\sigma_{1} p_{1}, \ldots \sigma_{n} p_{n}\right)=\sqrt{(n+1)} \int f(p) d \Omega_{p} \Psi^{(n+1)} \\
\left(p, \sigma ; p_{1} \sigma_{1}, \ldots p_{n} \sigma_{n}\right)
\end{gathered}
$$

The action of $\mathscr{P}$ on $h$ is given by

$$
(\mathscr{U}(a, \Lambda) \Psi)^{(n)}\left(\sigma_{1} p_{1} \ldots \sigma_{n} p_{n}\right)=\Psi^{(n)}\left(W(\Lambda) \sigma_{1}, \Lambda^{-1} p_{1} ; \ldots W(\Lambda) \sigma_{n}, \Lambda^{-1} p_{n}\right) .
$$

It is then easy to check that the transformation law and canonical commutation relations hold for $a(\Lambda, f)$ on $D$, and that $\mathscr{U}(a, \Lambda) D \subset D$.

We wish to define a causal field, that is, a map $A$ from $K_{0} \times S$ to unbounded operators, with the properties given in Section 1. Experience with finite-dimensional fields suggests that we should combine annihilation operators of particles with creation operators of anti-particles. Let $P_{1}$ be the injection $K_{0} \rightarrow K$ given by $P_{1} \alpha=(\alpha, o)$, that is, $P_{1} \alpha \in K_{1} \subset K$; and let $P_{2}$ be the injection $K_{0} \rightarrow K$ given by $P_{2} \alpha=(0, \alpha) \in K_{2} \subset K$. Then for any $\alpha \in K_{0}, a\left(P_{1} \alpha, f\right)^{*}$ is a creation operator for a particle, and $a\left(P_{2} \alpha, f\right)^{*}$ is the creation operator for an antiparticle (conventionally written $\left.b(\alpha, f)^{*}\right)$. Obviously we have

$$
W P_{i}=P_{i} W_{0}, \quad i=1,2 .
$$

We wish the map $A$ to be linear, and so we are led to introduce an antilinear map $B: K_{0} \rightarrow K_{0}$ (its further properties are determined by the requirements of causality and covariance). Then define the free field $A$ to be the map

$$
A(\alpha, f)=a\left(P_{1} \alpha, f\right)+a\left(P_{2} B \alpha, \bar{f}\right)^{*} ; \alpha \in K_{0}, f \in S .
$$

In order that $\mathscr{U}(\Lambda) A(\alpha, f) \mathscr{U}(\Lambda)^{-1}=A\left(W_{0} \alpha, f_{\Lambda}\right)$ it is necessary and sufficient that $B$ commute with $W_{0}(\Lambda)$. We see that $A(\alpha, f)$ always commutes with $A(\beta, g)$ since

$$
\left[a\left(P_{1} \alpha, f\right), a\left(P_{2} B \beta, g\right)^{*}\right]=\left(P_{\mathbf{2}} B \beta, P_{1} \alpha\right) \int \tilde{f}(p) \tilde{g}(-p) d \Omega_{p}=0
$$

and so forth. 
We find for $A$ and $A *$

$$
\begin{aligned}
& {\left[A(\alpha, f), A(\beta, g)^{*}\right]} \\
& \quad=\left[a\left(P_{1} \alpha, f\right), a\left(P_{1} \beta, g\right)^{*}\right]+\left[a\left(P_{2} B \alpha, \bar{f}\right)^{*}, a\left(P_{2} B \beta, \bar{g}\right)\right] \\
& \quad=\left(P_{1} \alpha, P_{1} \beta\right) \int \tilde{g}(-p) f(p) d \Omega_{p}-\left(P_{2} B \beta, P_{2} B \alpha\right) \int \tilde{g}(p) f(-p) d \Omega_{p} \\
& \quad=\int f(p) \tilde{g}(-p) \delta\left(p^{2}-m^{2}\right)\left[\vartheta\left(p^{0}\right)(\alpha, \beta)-\vartheta\left(-p^{0}\right)(B \beta, B \alpha)\right] d^{4} p .
\end{aligned}
$$

This is causal if and only if $(B \beta, B \alpha)=(\alpha, \beta)$ i.e. $B$ is anti-unitary. In the next section we show that for a certain unitary representation $W_{0}$ of $L \uparrow$ such a $B$ exists. Since the vacuum expectation values are obviously distributions, we have constructed a field in the sense of section 1. Its truncated functions are zero beyond the two-point functions which are

$$
\left\langle\mathrm{A}(\alpha, f)^{*} \mathrm{~A}(\beta, g)\right\rangle=(\alpha, \beta) \int \bar{f}(x) g(y) \Delta^{+}(x-y) d^{4} x d^{4} y \text {. }
$$

In order to show that there exists a corresponding theory of local observables, it is sufficient to repeat the argument of Borchers and ZimmermanN [9], which goes through with virtually no change. This completes the construction of the counter-example.

\section{Proof of Existence of $B$ for Certain Unitary Representations}

Consider the representation $W_{0}$ of $S L(2, C)$ that is induced [13] from the representation $\mathscr{D}^{\frac{1}{2}}=\mathscr{D}$ of $S U_{2} \subset S L(2, C)$. We denote by $\mathscr{M}$ the two dimensional carrier space of $\mathscr{D}$. We may identify the carrier space of $W_{0}$ with the space of functions $\psi: S L(2, C) \rightarrow \mathscr{M}$, square integrable with respect to Haar measure, that also satisfy the left-covariance condition

$$
\psi_{\alpha}(U A)=\mathscr{D}(U)_{\alpha \beta} \psi_{\beta}(A) \text { for all } U \in S U_{2}, A \in S L(2, C) .
$$

Call this space $K_{0}$. Now $\mathscr{D}$ is unitary equivalent to its complex conjugate representation, so there exists a unitary operator $V$ in $\mathscr{M}$ such that

$$
\overline{\mathscr{D}(U)_{\alpha \beta}}=\left(V^{-1} \mathscr{D}(U) V\right)_{\alpha \beta}
$$

where we have written $\mathscr{D}$ in $2 \times 2$ matrix form. Define the anti-linear $\operatorname{map} B: K_{0} \rightarrow K_{0}$ by

$$
(B \psi)_{\alpha}(A)=V_{\alpha \beta} \overline{\psi_{\beta}(A)} .
$$

This is well defined. For if $\psi$ is left covariant then

$$
\begin{aligned}
(B \psi)_{\alpha}(U A) & =V_{\alpha \beta} \overline{\psi_{\beta}(U A)}=V_{\alpha \beta} \overline{\mathscr{D}(U)_{\beta \gamma}} \bar{\psi}_{\gamma}(A) \\
& =\mathscr{D}(U)_{\alpha \beta} V_{\beta \gamma} \bar{\psi}_{\gamma}(A)=\mathscr{D}(U)_{\alpha \beta}(B \psi)_{\beta}(A) .
\end{aligned}
$$

Thus $B \psi$ also satisfies the left-covariance condition.

We easily check that $B$ is anti-unitary; it commutes with the action of $S L(2, C)$ on $K_{0}$, which is by right multiplication:

$$
\left(W_{0}\left(A_{1}\right) \psi\right)(A)=\psi\left(A A_{1}\right) \text {. }
$$

It is known that $W_{0}$, restricted to $S U_{2}$, is equivalent to the representation $V_{0}$ of $S U_{2}$ introduced earlier. This completes the proof. 
The representation $U$ of $L \uparrow$ chosen here is highly reducible, and so differs from that discussed in [2]. A similar discussion could have been given in their case.

Our representation $W_{0}$ happens to have the following property. Let $\left[m, \frac{1}{2}\right]$ be the representation of $\mathscr{P}$ with positive mass, energy and spin $\frac{1}{2}$. Then $W_{0}$ is the restriction of $\left[m, \frac{1}{2}\right]$ to the Lorentz group. To see this, note that for each $p, \mathscr{D}^{\frac{1}{2}}$ can be extended (in the same space) to the

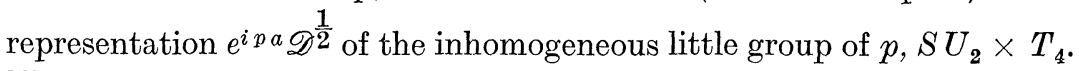
The resulting representation of $\mathscr{P}$, induced from $S U_{2} \times T_{4}$, is the same as the representation of $L \uparrow$ induced from $\mathrm{SU}_{2}$; this can be checked by explicit computation ${ }^{1}$.

Because of this, the representation $W$ in $K$ can be extended to a representation of $\mathscr{P}$ with mass $m_{0}$ (arbitrary) and spin $\frac{1}{2}$. Thus the states $\alpha \in K$ can be labelled according to the Wigner canonical form; namely, to each $\alpha \in K$ is given a wave-function $\alpha_{i}(\mathbf{q}), i=1,2$, with scalar product

$$
(\alpha, \beta)=\sum_{i} \int \frac{d^{3} q}{2 q_{0}} \bar{\alpha}_{i}(q) \beta_{i}(q), q_{0}=\sqrt{q^{2}+m_{0}^{2}}
$$

and transforming according to

$$
(W(\Lambda) \alpha)_{i}(\mathbf{q})=\mathscr{D}_{i j}\left(L_{q} \Lambda L_{\Lambda^{-1} q}\right) \alpha_{j}\left(\Lambda^{-1} \mathbf{q}\right) .
$$

We replace $\alpha_{i}(\mathbf{q})$ by a manifestly covariant wave function $\alpha_{i}(y)$, where

$$
\begin{aligned}
& \tilde{\alpha}_{i}(q)=\delta\left(q^{2}-m_{0}^{2}\right) \theta\left(q_{0}\right) D_{i j}\left(L_{\Lambda q}\right) \alpha_{j}(\boldsymbol{q}) \\
& \alpha_{i}(y)=\int e^{t g y} \tilde{\alpha}(q) d^{4} q .
\end{aligned}
$$

One may then define a bi-local field in terms of the field $A(\alpha, f)$. Any real test function $h(\mathrm{y}) \in \mathrm{S}\left(R^{3}\right)$ determines a unique real solution $\alpha(h)$ of the Klein-Gordon equation, for each $y_{0}$, by the conditions

$$
\begin{aligned}
& \alpha\left(\mathbf{y}, y_{0}\right)=h(\mathbf{y}) \\
& \dot{\alpha}\left(\mathbf{y}, y_{0}\right)=0 .
\end{aligned}
$$

This solution has a unique positive energy part. The bilocal field $\mathrm{A}_{i}\left(\mathbf{h}, y_{0} ; x\right)$ is then equal to $\mathrm{A}\left(\alpha_{i}(h), x\right)$, where $\alpha_{i}(h) \in K$ is the wave function with $\alpha(h)$ as its $i$ th component. The bilocal field transforms according to $\mathscr{U}(a, \Lambda) A_{i}(y, x) \mathscr{U}^{-1}(a, \Lambda)=D(\Lambda)_{i j} A_{\jmath}(\Lambda y, \Lambda x+a)$ where $D$ is the two-dimensional representation of $L \uparrow$ (two-valued).

Bi-local fields were introduced heuristically by Yukawa [14], and can be given [15] an axiomatic treatment similar to that of WigHTMAN. The field $A_{i}(y, x)$ constructed here is the spin $\frac{1}{2}$ version of the "free fields of infinite spin", defined in [16]. The present synthetic method

\footnotetext{
1 I am indebted to M. Froissart for this remark.
} 
has the advantage of showing clearly the reduction of $U(a, \Lambda)$ into irreducibles, that is, what multiplicity the various spins possess.

We may remark that a spin-statistics theorem can be proved in $S$-matrix theory [17]. It follows that the $S$-matrix of an interacting field satisfying the properties of our section 1, will not possess all the properties attributed to the $S$-matrix in [17].

The usual $S$-matrix axioms must contain information equivalent to the finiteness of the interpolating field. This is probably contained in the form assumed for crossing symmetry. Naturally, it is not obvious that the particles associated with an interacting field of the type discussed in this paper necessarily fall into an infinite multiplet all of the same mass. The field could have no particles associated with it, linearly. The particles, for example, might be created by bilinear combinations of the field, and be either finite in number or non-degenerate in mass.

It is interesting to speculate that the free fields defined here, as well as the "free fields of infinite spin" defined earlier [15] by the author, violate the compactness condition suggested by HAAG and SwIECA [18].

Acknowledgements. The author thanks P. T. Matrhews and G. Feldman for many discussions about their models, and C. Fronsdal for a prepublication copy of his ideas.

\section{References}

[1] Fronsdal, C.: Contribution to "High energy physics and elementary particles". International Atomic Energy Authority, Vienna 1966 (Proceedings of the 1965 Seminar at Trieste).

[2] Feldman, G., and P. T. Matthews: Unitarity, causality and fermi statistics. Phys. Rew. (to be published).

[3] Araki, H.: Local quantum theory. New York: W. A. Benjamin 1966.

[4] Borchers, H. J.: Commun. Math. Phys. 1, 281 (1965).

[5] Joos, H.: Fortschr. Physik 10, 65 (1962).

[6] Pursey, D. L.: Ann. Phys. 32, 157 (1965).

[7] Weinberg, S.: Phys. Rev. 133 B, 1318 (1964).

[8] Garding, L., and A. S. Wightman: Arkiv Fisik 28, (13), 129 (1965).

[9] Borchers, H. J., and W. Zimmermann: Nuovo Cimento 31, 1047 (1964).

[10] Mackey, G. W.: Appendix to: Mathematical problems of relativistic physics, by I. E. Segal. Am. Math. Soc. (1963).

[11] NaImaRK, M. A.: Linear representation of the Lorentz group. Oxford: Pergamon 1964.

[12] Cook, J. M.: Trans. Am. Math. Soc. 74, 222 (1953); R. F. Streater, and A. S. Wightman, PCT, spin and statistics and all that, p. 103. New York: W. A. Benjamin 1964.

[13] Hermann, R.: Lie groups for physicists. New York: W. Benjamin 1965.

[14] Yukawa, H.: Phys. Rev. 76, 300, 1731 (1949); 77, 219, 849 (1950).

[15] Streater, R. F.: Proc. Phys. Soc. 83, 549 (1964).

[16] - Ann. Phys. 30, 1 (1964).

[17] Lu, E. Y. C., and D. I. Olive: Nuovo Cimento 45 A, 205 (1966).

[18] HaAG, R., and J. SwIecA: Commun. Math. Phys. 1, 308 (1965). 